\title{
Loss of RPS27a expression regulates cell cycle, apoptosis, and proliferation via the RPS27a-RPL11- MDM2-p53 pathway in lung adenocarcinoma cells
}

Hongyan Li

Institute of Modern Physics Chinese Academy of Sciences

Hong Zhang ( $\sim$ Zhangh@impcas.ac.cn )

Institute of Modern Physics Chinese Academy of Sciences

Guomin Huang

Institute of Modern Physics Chinese Academy of Sciences

Zhitong Bing

Institute of Modern Physics Chinese Academy of Sciences

Hongtao Luo

Gansu Provincial Cancer Hospital

Duling Xu

Institute of Modern Physics Chinese Academy of Sciences

Jiadi Liu

Institute of Modern Physics Chinese Academy of Sciences

Xiaoli An

Institute of Modern Physics Chinese Academy of Sciences

\section{Research Article}

Keywords: Ribosomal protein S27a (RPS27a), Lung adenocarcinoma, Apoptosis

Posted Date: August 3rd, 2021

DOI: https://doi.org/10.21203/rs.3.rs-757515/v1

License: (c) (i) This work is licensed under a Creative Commons Attribution 4.0 International License.

Read Full License

Version of Record: A version of this preprint was published at Journal of Experimental \& Clinical Cancer Research on January 24th, 2022. See the published version at https://doi.org/10.1186/s13046-02102230-z. 


\section{Abstract \\ Background}

Some ribosomal proteins (RPs) might regulate the MDM2-p53 loop by binding to RPL5 or RPL11. This study aimed to explore whether ribosomal protein S27a (RPS27a) interacted with the ribosomal protein L11 (RPL11) to regulate p53 in lung adenocarcinoma (LUAD) cells.

\section{Methods}

RPL11-interacting proteins were identified using a proteomics approach. Co-immunoprecipitation (co-IP), docking analysis, GST-fusion and in vitro ubiquitination assay were used to analyze the interaction of RPS27a and RPL11. Cell cycle, apoptosis, cell invasion, cell viability and colony-formation assay were analyzed by knocking down RPS27a. The RPS27a mRNA expression in LUAD was analyzed based on the TCGA dataset and the RPS27a expression was detected by immunohistochemistry in LUAD samples. At last, the RPS27a and p53 expression were analyzed by immunohistochemistry in xenograft tumors by blocking RPS27a.

\section{Results}

The ablation of RPS27a inhibits murine double minute 2 (MDM2)-mediated p53 ubiquitination, induced G1/S cell cycle arrest and apoptosis, and inhibited the proliferation of LUAD cells. Also, it induced p53dependent cell cycle arrest and RPL11-dependent p53 activation. The protein-protein docking results revealed that RPS27a and RPL11 formed a stable complex structure. The GST-fusion protein-protein association assay demonstrated that RPS27a bound to RPL11. The overexpressed RPS27a in LUAD was found to be correlated with a poorer prognosis based on the TCGA dataset. RPS27a expression was high in patients with LUAD. Blocking RPS27a increased p53, thus, suppressing cell proliferation and A549 xenograft growth in nude mice.

\section{Conclusions}

This study was novel in reporting that RPs bound to RPL11 to regulate the MDM2-p53 feedback loop, revealing that RPS27a plays an important function in LUAD growth. Hence, RPS27a might provide a diagnostic marker or therapeutic target for patients with LUAD.

\section{Background}

China ranks first in terms of lung cancer incidence worldwide [1, 2]. Lung adenocarcinoma (LUAD) is the main histological subtype of lung cancer [3], and a 5-year overall survival rate of LUAD is less than $20 \%$ $[4,5]$. The mechanism of LUAD development is complex, and the effect of oncogenes on LUAD is still 
unknown [6]. Moreover, as a tumor suppressor, p53 expression is inhibited and cannot exert a transcriptional activation effect in LUAD [7]. However, the stability and activation of p53 are mainly regulated by the murine double minute 2 (MDM2), which constitutes MDM2-p53 feedback loop necessary in regulating apoptosis [8].

New discoveries have been made about the regulation of the MDM2-p53 loop in recent years. Under nucleolar stress, some ribosomal proteins (RPs), which constitute ribosomal subunits, freely enter into the nucleoplasm without being degraded by the proteasome [9]. Further, they directly or indirectly bind to MDM2, which then stabilizes and activates p53 [10]. Therefore, a variety of RPs can bind to MDM2, form an "RPs-MDM2-p53" complex, such as RPS7 [11], RPL5 and RPL11 [12], and RPL26 [13] transfer into the nucleoplasm, and then regulate the p53 activity. Some RPs, including RPS6 and RPL22 [14], RPL24 [15], RPS19 [16], RPS14 [17], and RPL4 [18], activating MDM2-p53 loop are RPL11 and RPL5 dependent because RPL11 and RPL5 are nucleolar stress effectors and sensors. The RPL5/RPL11-MDM2-p53 complex is the most classic model of interaction between RPs and p53 [12]. RPL5 and RPL11 act alone with MDM2 or form a 5S ribonucleoprotein complex with 5S rRNA [19]. The role model of RPS14 and RPL4 with MDM2-p53 was as follows. The reduced expression of RPS14 and RPL4 inhibited MDM2 activity through the binding of RPL5/RPL11-MDM2 pathway, and led to p53 accumulation $[17,18]$. Based on previous findings, it was concluded that some RPs might regulate the MDM2-p53 loop by binding to RPL5 or RPL11 and form the RPs-RPL5/RPL11-MDM2-p53 pathway.

Hence, it was believed that other RPs bound to RPL5 or RPL11 to regulate the MDM2-p53 loop. In this regard, immunoprecipitated proteins (IP)/Mass spectrometry (MS) were used to identify the RPs interacting with RPL11. Among the identified RPs, the ribosomal protein S27a (RPS27a) gained attention. The present study found that high expression of RPS27a might be associated with the development of LUAD. The knockdown of RPS27a generated ribosomal stress that induced p53-dependent cell cycle arrest and apoptosis, which required RPL11. Further investigation indicated that RPS27a bound to RPL11 and regulated the MDM2-p53 loop, and was a regulator of the RPL11-MDM2-p53 pathway in LUAD.

\section{Methods}

\section{Cell culture}

We prepared four cell lines (BeNa, Culture Collection, Beijing, China), BEAS-2B (The human bronchial epithelial cells, no.BNCC254518), H460 (The human large cell lung cancer cell line, no. BNCC233991), A549 (The human non-small-cell lung cancer cell lines, no. BNCC290808) and H1299 (The human nonsmall-cell lung cancer cell lines, no. BNCC334400), they cultured in F-12K medium (Hyclone, MA, USA) with $10 \%$ fetal bovine serum (Gibco, CA, USA) and kept under $37^{\circ} \mathrm{C}$ with $5 \% \mathrm{CO}_{2}$. Follow-up experiments were performed when the cells were in the logarithmic phase of growth and $80 \%$ confluence.

\section{IP/MS}


The immunoprecipitation of RPL11 was performed as described previously [20]. Briefly, $10 \mu \mathrm{g}$ of rabbit RPL11 antibodies (Abcam, Cambridge, UK) and the same amount of rabbit IgG (no. P2173, beyotime, Shanghai, China) were added in A549 cell lysate of the experimental and control groups, respectively, followed by overnight incubation at $4^{\circ} \mathrm{C}$. After elution and purification, the immunoprecipitates were separated, then stained with silver. The immunoprecipitation was digested and then analyzed using an LC-MS/MS (TripleTOF, AB Sciex, Boston, MA, USA) instrument, and the results were evaluated. Credibility $\geq 95 \%$ and unique peptides $\geq 1$ were the criteria to identify proteins [21].

\section{Plasmids, drugs, antibodies, and siRNAs}

His-tagged RPL11 expression plasmids were constructed by inserting the RPL11 cDNA into the PET32a His vector at $\mathrm{Ncol}$ and $\mathrm{Xhol}$ sites (GE Healthcare, Boston, MA, USA) (Supplementary File 1). The RPL11 cDNA was amplified using the following mRNA primers: $5^{\prime}-$

GACGACGACAAGGCCATGGCTGCGCAGGATCAAGGTG-3' and 5'-

GTGGTGGTGGTGGTGCTCGAGTTTATTTGCCAGGAAGGATG-3'. A GST-RPS27a Escherichia coli

expression vector was constructed by inserting the RPS27a cDNA into the pEGX.6P.1 vector at BamHI and Xhol sites (GE Healthcare) (Supplementary File 1). The RPS27a cDNA was amplified using the following mRNA primers: 5'-GTTCCAGGGGCCCCTGGGATCCATGCAGATTTTCGTGAAAAC-3' and 5'-

CAGTCACGATGCGGCCGCTCGAGTTACTTGTCTTCTGGTTTG-3'. The Flag-RPS27a and His-RPL11 were also generated using polymerase chain reaction (PCR) and cloned into the pIRES2-ZsGreen1 vector.

Lipo2000 (no. 11668019, Invitrogen, CA, USA) was used for RNAi experiments. $\beta$-actin (no. ab8227), RPS27a (no. ab74731), p53 (no. ab74731), p21 (no. ab109199) (Abcam, Cambridge, UK), and MDM2 (no. ab16895) (Genetex, NJ, USA) were used for immunoblotting (IB). MG132 (no. HY-13259) was purchased from Medchemexpress (NJ, USA), Doxorubicin (Dox, no. GC16994) and Actinomycin D (ActD, no. GC16866) were purchased from Glpbio (CA, USA). Three different siRNAs of each gene was synthesized by Genepharma (St. Louis, Shanghai, China); their sequences were shown in Supplementary File 2.

\section{GST-fusion assay}

His-tagged RPL11 expression plasmids were transfected in Escherichia coli BL21. The His-tagged RPL11 was purified using an $\mathrm{Ni}^{2+}{ }^{+}$NTA column (Qiagen, Shanghai, China) after expression in E. coli. The GSTfusion assay was conducted as previously described. Then, $50 \mu \mathrm{g}$ GST-RPS27a or GST was mixed with glutathione Sepharose 4B beads (Sigma) and incubated with $20 \mu \mathrm{g}$ purified His-RPL11 proteins. anti-STag and GST antibodies were used to analyze protein interactions by IB [22].

\section{Transient transfection, IB, and co-immunoprecipitation (co- IP) analyses}

A549 cells were inoculated in six-well plates and cultured in complete medium. When the cells grew to $70 \%$ confluence, the cells were transfected with siRNA. The transfected cells for $48 \mathrm{~h}$ were collected and treated with lysis buffer. Equal amounts of clear cell lysate $(50 \mathrm{mg})$ were used for IB and co-IP analyses [23]. 


\section{Immunofluorescence}

The immunofluorescence assay was performed as described previously [20]. Cells were then scanned and analyzed using a confocal laser microscope (LSM, Carl Zeiss AG, Germany).

\section{Cell cycle and apoptosis analysis}

After $48 \mathrm{~h}$ of transfection, the analysis of cell cycle and apoptosis was performed as

described previously [21, 22]. Data of DNA content were collected using CellQuest and analyzed using a ModFit software program. The apoptotic cells were analyzed with a Flowsight imaging flow cytometer (Amnis/Merck Millipore, Darmstadt, Germany).

\section{In vitro ubiquitination assay}

In vitro ubiquitination experiments refer to previous studies using $\mathrm{Ni}^{2+}{ }^{+} \mathrm{NTA}$ purification method [24]. The $\mathrm{Ni}^{2+}$-NTA pulldown used for ubiquitination experiment, the bead-bound proteins were analyzed using IB.

\section{Reverse transcription and quantitative PCR analyses}

TaKaRa company designed and synthesized primers (Dalian, China), andtwo-step

SYBR kit to complete real-time fluorescent quantitative PCR on the FTC-3000 qPCR system (Stratagene, California, USA). The primers used were 5'- AGAAGAAGTCTTACACCACTCCC-3' and 5'-

TGCCATAAACACCCCAGC-3' (RPS27a); 5'-TCCACTGCACAGTTCGAGGG-3' and 5'-

AAACCTGGCCTACCCAGCAC-3' (RPL11); 5'- CGACTGTGATGCGCTAATGG-3' and 5'-

AAATCTGTCAGGCTGGTCTGC-3' (p21); 5'-CTCACCATCATCACACTGGAA-3' and 5'-TCATTCAGCTCTCGG AACATC-3' (p53); 5'-AATCATCGGACTCAGGTACATC-3' and 5'-CTGCTACTGCTTCTTTCACAAC-3' (MDM2); 5'-TCAAGAAGGTGGTGAAGCAGG-3' and 5'-TCAAAGGTGGAGGAGTGGGT - 3' (GAPDH) [25].

\section{Dissociation of ribosomal subunits and measurement of the subunit ratio}

The dissociation of ribosomal subunits was performed as described previously [26]. Samples measured at $254 \mathrm{~nm}$ absorbance (Biocomp, CA) and quantitative analysis of ribosome peaks. The area under the curve from the lowest point to the lowest point of the $40 \mathrm{~S}, 60 \mathrm{~S}$, and $80 \mathrm{~S}$ peaks was calculated by summing the digital measurements.

\section{Stably knockdown of RPS27a cells constructed}

A stable knockdown of the RPS27a (RPS27a knockdown) cell line was achieved by lentiviral infection and drug screening. In brief, a $7.5 \times 10^{4}$ cells $/ \mathrm{mL}$ suspension was made from $A 549$ cells, and $2 \mathrm{~mL}$ of the suspension per well was seeded in a six-well plate. The virus was added $20 \mathrm{~h}$ after seeding the cells. The fresh medium was replaced with $2 \mu \mathrm{g} / \mathrm{mL}$ puromycin every $2-3$ days.. 
Transwell chambers (Costar, USA were used for Transwell invasion assays, as previously described [27]. The $\mathrm{IC}_{50}$ of doxorubicin (Dox) on A549 cells and its effect on the survival of A549 cell clones are shown in Supplementary File 3. The incubation was continued for $24 \mathrm{~h}$. The cells were fixed with $4 \%$ paraformaldehyde and stained with crystal violet solution..

\section{Cell viability assay}

The cell counting kit 8 (CCK-8, B34304, Bimake, USA) was used to detect cell proliferation. A microplate reader (Tecan M200, Switzerland) was used to measure the absorbance at a wavelength of $450 \mathrm{~nm}$. The formula, Cell viability $(\%)=[\mathrm{A}($ compound +$)-\mathrm{A}$ (blank)]/[A (compound -$)-\mathrm{A}($ blank $)] \times 100 \%$ was used to calculate the growth percentage. Cell viability means cell proliferation [27].

\section{Colony-formation assay}

The colony-formation assay of A549 cells was performed as described previously [28]. The colony formation rate was calculated by dividing the number of colonies/number of cells $\times 100 \%$ by the control.

\section{Docking analysis}

Briefly, the protein-protein interaction module of Schrodinger software (Schrodinger 2015 suit) was used for analyzing RPL11 and PRS27a interactions. The three-dimensional crystal structures of the human 80 s ribosome (PDBID: 4v6x) were extracted from the PDB database. The small-molecule 3D structures were docked from the x-ray crystal structure of RPL11 and PRS27a, and two proteins were extracted from the $80 \mathrm{~S}$ ribosome. The ubiquitin and water molecules were removed from the two protein structures to simulate the interaction [29].

\section{Tumor xenografts}

The management and handling of animals complied with the administrative regulations of the Laboratory Animal Affairs Administration of the Ministry of Science and Technology of China (1988.11.14). The research on experimental animals was approved by the Ethics Committee of the Institute of Modern Physics, Chinese Academy of Sciences, and the Institutional Animal Care and Use Committee. Four-five-old nude mice (female, weight 16-17 g, SPF level) were obtained from the Laboratory Animal Center of obio biotechnology (Shanghai, China). NC and RPS27a knockdown cells in the logarithmic growth phase were injected $\left(2 \times 10^{6}\right)$ subcutaneously into mice to establish a cell xenograft model. Based on the equation of the flat spheroid [tumor volume $=(\text { short diameter })^{2} \times$ large diameter $\times \pi / 6]$, the average tumor volume of each group was calculated and expressed in $\mathrm{mm}^{3}[30]$.

\section{Immunohistochemistry analysis}

The LUAD samples and xenograft tumors were analyzed by immunohistochemistry was performed as described previously [30]. The sections were examined with an 80i Nikon optical microscope (Nikon, Tokyo, Japan). Image-Pro Plus software was also used to analyze the optical density of protein expression. 


\section{Statistical analysis}

Prism 8 software (GraphPad Software, CA, USA) was used to analyze the data. Statistical differences were analyzed based on the Student's t test and on one-way analysis of variance test. $p$ value of $<0.05$ was represented statistically significant.

\section{Results}

\section{RPS27a is a potential binding protein with RPL11}

The untreated A549 cell lysate was used to identify endogenous RPL11-binding proteins by IP/MS to discover the potential RPL11-binding ribosomal proteins. The silver staining image of the binding proteins showed that a band was detected at about $18 \mathrm{kDa}$ (Fig. 1A). The number of proteins identified in the IP protein sample of the band was 133. The protein-related information is shown in Supplementary File 4, among which 43 interactors were RPs (Fig. 1B). The combined degree of RPS27a was the highest. Next, we detected the expression of RPS27a in BEAS-2B, A549 and H460 cells (Fig. 1C), the results showed in expression of RPS27a in H460 and A549 cells was higher than BEAS-2B cell, and was highest in A549 cell (Fig. 1D). This results indicated that the high expression of RPS27a is associated with the tumor growth of non-small cell lung cancer. Moreover, a previous study showed the RPS27a was involved in the regulation of p53 levels. Therefore, the present study focused on the hypothesis that the RPS27aRPL11 interaction might involve in p53 activation.

\section{Correlation of A549 cell apoptosis with RPS27a expression}

Inducing tumor cell apoptosis is a common strategy to inhibit tumor development. We have demonstrated that carbon ion radiation (CIR) induced nucleolar stress leads to reduction of RPL27a expression and promote spermatogonia apoptosis. Therefore, $4 \mathrm{~Gy}$ CIR were used to induce apoptosis of A549 cells. Then, the apoptosis of A549 induced by CIR (Fig. 1E) and expression of RPS27a was detected (Fig. 1G). The results showed that the increased apoptotic ratio (Fig. 1F) and the decreased expression of RPS27a were time dependent (Fig. 1H). Therefore, the A549 cell apoptosis has correlation with the reduction of RPS27a expression.

\section{Knockdown of RPS27a activated p53, promoted cell apoptosis, and inhibited cell proliferation}

Small RNA interference and overexpression plasmids were used to knock down and induce overexpression of RPS27a, respectively, so as to explore the relationship between RPS27a and RPL11, and p53 activation. The protein levels of p53, MDM2, p21, RPL11 and RPS27a increased in RPS27a siRNA-treated cells compared to NC cells (Fig. 2A). Similar to the immunoblotting results, the immunofluorescence results also showed that the fluorescence signal of RPL11 (Fig. 2B) was enhanced in the nucleus and cytoplasm after knocking down RPS27a (Fig. 2C). The mRNA expression levels of 
genes increased in knockdown of RPS27a cells compared to NC cells (Fig. 2D). In addition, the knockdown of RPS27a promoted cell apoptosis, inhibited cell proliferation and accelerated G1/S cell cycle progression (Fig. 2E-G). Moreover, the increased p53 expression was relatively stable with an increased half-life (Fig. 2H). The aforementioned findings suggested that the knockdown of RPS27a stabilized and activated p53, resulting in A549 cell apoptosis. Representative images of the flow cytometer are shown in Figures S1 and S2.

\section{Knockdown of RPS27a induced nucleolar stress}

The immunofluorescence technology was used to analyze the distribution of nucleolar integrity marker proteins, nucleolin (NCL) (Fig. 2J) and nucleophosmin (B23) (Fig. 2K) to further determine whether RPS27a knockdown would destroy the nucleolar structure of A549 cells. The results showed that RPL27a knockdown induced the movement of NCL and B23 into the nucleoplasm, indicating that the nucleolar stress was generated. Further, polysome profiles were compared to study the effect of RPS27a knockdown on the ratios between small and large ribosomal subunits in A549 cells (Fig. 2L). The 80S:60S ratio decreased and 80S:40S increased was observed in the RPS27a knockdown cells. Thus, the knockdown of RPS27a impaired the ribosomal profiles in A549 cells, which might be related to the changes in the nucleolus. These results indicated that RPS27a knockdown could change the ribosomal subunit ratio and generate nucleolar stress.

\section{Knockdown of RPS27a induced RPL11-dependent p53 activation and p53-dependent cell cycle arrest}

The co-transfection experiment of RPS27a and p53 showed that the knockdown of p53 eliminated the increase in MDM2 and p21 protein levels (Fig. 3A), G1-phase arrest (Fig. 3B), and apoptosis (Fig. 3C) induced by the knockdown of RPS27a. In addition, the knockdown of p53 eliminated the inhibition of cell growth and proliferation (Fig. 3D). In H1299 cells, the knockdown of RPS27a inhibited cell proliferation (Fig. 3D) and induced G1 phase arrest (Fig. 3B). Representative images of the flow cytometer are shown in Figures S3 and S4.

Deleting some ribosomal proteins induces ribosomal stress to activate $\mathrm{p} 53$, which is mainly mediated by RPL11. The RPS27a and RPL11 co-transformation experiments were performed to prove that knocking down RPS27a to activate p53 was also regulated by this mechanism. The results showed that the knockdown of RPL11 eliminated the increase in MDM2 and p21 protein levels (Fig. 3E), G1-phase arrest (Fig. 3F), and apoptosis (Fig. 3G) induced by the knockdown of RPS27a. In addition, the knockdown of RPL1 1 eliminated the inhibition of cell growth and proliferation (Fig. 3H). Representative images of the flow cytometer are shown in Figures S5 and S6. Therefore, the knockdown of RPS27a requires RPL11 to induce p53 upregulation and a decrease in cell proliferation and growth.

\section{Knockdown of RPS27a stabilized p53}


In addition, the co-immunoprecipitation assay showed that the knockdown of RPS27a enhanced the binding interaction between RPL11 and MDM2 (Fig. 4A). Therefore, the reduction of RPS27a was likely to enhance the interaction between RPL11 and MDM2, thereby inhibiting MDM2 and stabilizing p53. The A549 cells transfected with plasmid pIRES2-ZsGreen1-Hum-Ub-His and knocked-down RPS27a were constructed to analyze p53 ubiquitination to demonstrate that the RPS27a interacts with RPL11 and stabilizes $p 53$. Figure 4B shows that RPS27a partially rescued the degradation of $p 53$, and the expression of MDM2 and p53 increased in the combinations of His-Ub and knockdown of RPS27a, suggesting that RPS27a knockdown inhibited MDM2-mediated degradation of p53.

\section{RPS27a interacted with RPL11}

The small-molecule 3D structures were docked from the x-ray crystal structure of the RPS27a and RPL11 (Fig. 4C). RPL11 and PRS27a interactions were simulated using the protein-protein interaction module of Schrodinger software (Schrodinger 2015 suit). The 3D crystal structures of the human 80 S ribosome (PDBID: 4v6x) were extracted from the PDB database. The structure and function of proteins were closely related to the hydrogen bonding between amino acids. The well-known nucleotide-binding residues are shown in (Fig. 4C).

His-RPL11 and GST-RPS27a deletion fusion proteins purified from bacteria to further prove that RPS27a directly interacted with RPL11 in vitro. Figure 4D shows that purified Tag-RPL11 and purified Tag-MDM2 were bound by purified GST-RPS27a protein, but not GST alone. These results demonstrated that RPS27a directly bound to RPL11 in cells.

\section{Knockdown of RPS27a increases the sensitivity of A549 cells to actinomycin D (ActD) and doxorubicin (Dox) treatment}

The expression of proteins were detected in RPS27a-siRNA and then treated with ActD cells (Fig. 5A). p53 had similar half-life changes in control siRNA- and RPS27a siRNA-treated cells after $24 \mathrm{~h}$ of ActD treatment (Fig. 5B). The knockdown of RPS27a had the least effect on the level and stability of p53 protein (Fig. 5C). These results indicated that under the condition of nucleolar stress, the effect of RPS27a on p53 might be masked by other RPs.

This study evaluated the effect of knocking down RPS27a on DNA damage-induced p53 activation. The selection of Dox concentration was shown in Supplementary File 4. Figure 5D shows that the knockdown of RPS27a did not affect Dox-induced p53 activation. The half-life measurement showed that p53 induced small difference in control siRNA- and RPS27a siRNA-treated cells after $24 \mathrm{~h}$ of Dox treatment (Fig. 5E, F). The PCR results showed that knockdown of RPS27a had the least effect on the level of p53 target genes MDM2 and p21 (Fig. 5G). Therefore, these observations indicated that RPS27a was not involved in DNA damage-induced p53 stabilization. In addition, the study also analyzed cell apoptosis and cycle. The knockdown of RPS27a aggravated the Dox-induced G1 phase arrest (Fig. 5H). It also aggravated the suppression of the colony-forming (Fig. 5I, J), invasion (Fig. 5K, L), and migration abilities (Fig. 5M, N) in A549 cells. 


\section{RPS27a is a oncogene in LUAD}

The RPS27a mRNA expression in LUAD was analyzed based on the TCGA dataset to determine the role of RPS27a expression in the tumorigenesis of LUAD. The expression of RPS27a showed a significant difference between 483 LUAD tissues and 59 normal tissues (Fig. 6A), but no difference in four different LUAD stages (Fig. 6B). More dismal overall survival with high RPS27a expression of LUAD patients showed in the Kaplan-Meier survival analysis (Fig. 6C) and disease-free survival (Fig. 6D). More dismal survival with high RPS27a expression of LUAD patients showed in the plotter survival analysis (Fig. 6E). A total of 16 LUAD specimens and 10 normal tissue clinical specimens were collected from Gansu Cancer Hospital to determine the correlation of RPS27a expression in clinical LUAD. Representative images of immunohistological analysis are shown in Fig. 6F. As shown in Fig. 6G, the RPS27a expression were significantly upregulated in LUAD tissues compared with normal tissues. The findings proved for the first time that the overexpression of RPS27a in patients with LUAD might contribute to the development and short survival of LUAD.

The stable knockdown of RPS27a cells were injected into the left forelimb muscle of BALB/c nude mice to explore the effects of RPS27a in cell proliferation and apoptosis, and tumor nodules were harvested 53 days after injection (Fig. 7A). Silencing RPS27a inhibited tumor growth in vivo (Fig. 7B, C). It showed a relatively weak intensity of RPS27a, MMP-9, Ki67, and E-cadherin staining in knockdown of RPS27a cells (Fig. 7D-K), and a strong intensity of p53 in knockdown of RPS27a cells (Fig. 7L, M). These results indicated that p53 increased apoptosis by blocking RPS27a, and resulted in the inhibition of A549 xenograft growth in nude mice.

\section{Discussion}

RPS27a is a ribosomal protein constituting the 40S small subunit of the ribosome and plays an important role in ribosome biogenesis [31] and overexpressed in chronic myeloid leukemia, colon, renal, breast cancers and lung adenocarcinoma [32]. This study found that ablation of RPS27a expression could induce cell cycle arrest and apoptosis of A549 cells, which indicated that RPS27a is involve in regulator of the RPL11-MDM2-p53 pathway. The knockdown of RPS27a increased the expression of RPL11, promoted the binding of RPL11 to MDM2, leading to p53 activation. Therefore, RPS27a was a key factor in maintaining the normal level of p53 in LUAD. Also, it was crucial in negatively regulating the apoptosis of LUAD.

p53 is critical for regulating cell apoptosis and proliferation [33-35]. The activation of p53 is strictly regulated by its target gene product, an E3 ubiquitin ligase MDM2, forming an MDM2-p53 feedback loop $[36,37]$. Previous studies have shown that RPs can regulate p53 activation by inhibiting MDM2 activity, thereby affecting cell cycle progression and apoptosis [38]. This process is involved in regulating MDM2 binding by RPs to indirectly affect the negative feedback loop of MDM2-p53 [39]. Overexpressed RPs have similar functions as RPL11 and RPL5. They can bind to the central acid domain of MDM2, such as RPL23 and RPL26 [13], and RPS7 [11], which is followed by the inhibition of MDM2-mediated p53 
ubiquitination and degradation. In fact, some low-expressed RPs, such as RPL22 [14], RPS19 [16], RPS14 [17], and RPL4 [18] can also activate p53. This process must be completed with the participation of RPL11 and RPL5 [19]. The RPL5/RPL11-MDM2-p53 ternary complex was the most classic model of RPs and $\mathrm{p} 53$ binding because RPL 11 and RPL 5 were nucleolar stress effectors and sensors; RPL5 and RPL11 could bind to MDM2 alone or to MDM2 [19]. Moreover, 5S rRNA formed the 5S ribonucleoprotein complex (5S RNP) to bind to MDM2. Many RPs indirectly induced p53 activation through RPL11 and RPL5 [40]. Therefore, the combination of RPs with RPL11 and RPL5 cannot be ignored. The low-expressed RPS27a may have the aforementioned function. RPS27a have been found overexpressed in renal, breast and colon carcinomas [41, 42], a dramatic increase in the expression of RPS27a gene in an oncomouse model of hepatocellular carcinoma [43], it is essential role in the activation of cellular checkpoints via p53.

The cell cycle arrest and apoptosis caused by RPS27a knockdown were found to be RPL11 and p53 dependent. Co-transfection experiments proved that the knockdown of p53 eliminated the apoptosis and cell cycle arrest caused by the decreased expression of RPS27a, which was also observed in H1299 cells lacking p53, indicating that the apoptosis and cell cycle arrest caused by knocking down RPS27a was p53 dependent. In addition, the knockdown of RPS27a to activate p53 was RPL11 dependent because the knockdown of RPL11 eliminated the p53 activation caused by the knockdown of RPS27a, and attenuated cell cycle arrest and apoptosis induced by RPS27a knockdown.

This study found that the knockdown of RPS27a enhanced the binding of MDM2 and RPL11, and leading to the accumulation and activation of p53. The protein structures of RPL11 and RPS27a were constructed using homology modeling methods. The analysis of the results of protein-protein docking revealed that RPS27a and RPL11 formed a stable composite structure. GST experiments also confirmed that RPS27a had a binding relationship with RPL11. Taken together, these results suggested that RPS27a bound to RPL11, forming a complex with p53.

Studies exploring the role of RPS27a in stress-induced p53 activation have shown that RPS27a expression was regulated by the DNA damage response. ActD treatment showed that the knockdown of RPS27a had minimal effects on p53 stability (Fig. 5A-C). Although knocking down RPS27a could regulate p53 transcriptional activity in response to ribosomal stress, this effect was limited. Many nucleolar resident proteins were released to the nucleoplasm because the low dose of ActD destroyed the nucleolus and induced nucleolar stress. Therefore, the effect of RPS27a on p53 activity might be masked by other ribosomal proteins with the same effect. Dox treatment showed that the knockdown of RPS27a did not affect Dox-induced p53 activation, but had an impact on the degree of p53 activation. The half-life measurement showed that the half-life of p53 was similar in control and RPS27a siRNA-treated cells after $24 \mathrm{~h}$ Dox treatment (Fig. 5D-F). Therefore, the results indicated that RPS27a did not participate in DNA damage-induced p53 activation. The aforementioned results were different from previous findings showing that RPS26 regulated p53 transcriptional activity under DNA damage [44]. This discrepancy needs further investigation. 
This study found that the knockdown of RPS27a changed the ribosomal subunit ratio and induced nucleolar stress. It also enhanced the RPL11 expression and interaction between RPL11 and MDM2. This might be because the disruption of small subunits of ribosomes led to an enhanced translation of RPL11 through a 5'-top-mediated mechanism [45]. The knockdown of RPS27a caused the upregulation of RPL11 mRNA expression, thus, proving the aforementioned speculation.

RPS27a also had the ability to bind to MDM2, and inhibited the activity of MDM2 and leading to p53 stabilization [46]. In addition, RPS27a was not only an inducer of p53 but also a direct transcription target of p53 [47]. The DNA damage response induced the overexpression of RPS27a to active p53 [47]. In this study, another important pathway by which RPS27a regulated p53 activation was added, namely, RPS27a-RPL11-MDM2-p53. Together with RPS27a-MDM2-p53, the study provided the two modes of action of RPS27a in regulating p53. This study and previous studies together confirmed that nucleolar stress was monitored by an RPs-RPL5-RPL11-mediated p53 surveillance system.

\section{Conclusions}

In summary, this study showed that RPS27a is a new regulator of the RPL11-MDM2-p53 complex. This study was novel in demonstrating that RPs bound to RPL11 to regulate the MDM2-p53 feedback loop (Fig. 8). This study confirmed that the RPS27a-RPL11-MDM2-p53 signaling pathway was important in regulating the growth of LUAD cells, suggesting that RPS27a might be a potential target in LUAD treatment.

\section{Abbreviations}

4, 6-diamidino-2-phenylindole (DAPI); Ribosomal proteins (RPs); Phosphate buffered saline (PBS); polyvinylidene difluoride membrane (PVDF); Real-time quantitative polymerase chain reaction (PCR).

\section{Declarations}

\section{Ethics approval and consent to participate}

Written informed consent was obtained from patients with approval by the Institutional Review Board in Gansu Provincial Cancer Hospital and conducted in accordance with the principles of the World Medical Association Declaration of Helsinki. All animal experiments were approved by the Ethics Committee of the Institute of Modern Physics, Chinese Academy of Sciences, and conducted according to the Guidelines for the Care and Use of Animals for Scientific Research.

\section{Consent for publication}

All authors have read and agreed to publish this manuscript.

\section{Availability of data and materials}


The datasets used and/or analyzed during the current study are available from the corresponding author on reasonable request.

\section{Competing interests}

The authors declare that they have no competing interests.

\section{Funding}

This research was funded by the National Key R\&D Program of China (2018YFE0205100), the National Natural Science Foundation of China $(11875061,11505244)$, the National Natural Science Foundation of Gansu province (20JR5RA550), the National Laboratory of Heavy ion Accelerator of Lanzhou (EOHIRFL03P), and the Key Program of the National Natural Science Foundation of China (U1632270).

\section{Authors' contributions}

HYL conceived and designed the experiments, and drafted the manuscript. HZ conceived of the study, and participated in review and editing the manuscript. GMH and DLX performed the statistical analysis. ZTB and XLA performed the docking analysis. JDL and DLX helped with xenograft tumors experiments. HTL collected the clinical samples and information. All authors read and approved the final manuscript.

\section{Conflict of interest}

The authors declare no conflict of interest.

\section{Acknowledgements}

Not applicable.

\section{Author details}

${ }^{1}$ Department of Medical Physics, Institute of Modern Physics, Chinese Academy of Sciences, Lanzhou 730000, China. ${ }^{2}$ Key Laboratory of Heavy Ion Radiation Biology and Medicine of Chinese Academy of Sciences, Lanzhou 730000, China. ${ }^{3}$ Key Laboratory of Basic Research on Heavy Ion Radiation Application in Medicine, Lanzhou 730000, China. ${ }^{4}$ University of Chinese Academy of Sciences, Beijing, 100039, China. ${ }^{5}$ Department of Computational Physics, Institute of Modern Physics, Chinese Academy of Sciences, Lanzhou 730000, China. ${ }^{6}$ Department of Radiation Medicine, Institute of Modern Physics, Chinese Academy of Sciences, Lanzhou 730000, China. ${ }^{7}$ Radiation Oncology of Gansu Provincial Cancer Hospital, Lanzhou 730030, China.

\section{References}

1. Siegel RL, Miller KD, Jemal A. Cancer statistics 2016. CA Cancer J Clin. 2016;66:7-30. 
2. Hong QY, Wu GM, Qian GS, Hu CP, Zhou JY, Chen LA, et al. Lung Cancer Group of Chinese Thoracic Society; Chinese Alliance Against Lung Cancer. Prevention and management of lung cancer in China. Cancer. 2015; 121 Suppl: 3080-3088.

3. Chen Z, Fillmore CM, Hammerman PS, Kim CF, Wong KK. Non-small-cell lung cancers: a heterogeneous set of diseases. Nat Rev Canc. 2014;14:535-46.

4. Hirsch FR, Scagliotti GV, Mulshine JL, Kwon R, Curran WJJr, Wu YL, et al. Lung cancer: current therapies and new targeted treatments. Lancet. 2017;389:299-311.

5. Fry WA, Phillips JL, Menck HR. Ten-year survey of lung cancer treatment and survival in hospitals in the United States: a national cancer data base report. Cancer. 1999;86:1867-76.

6. Qiu M, Xu Y, Wang J, Zhang E, Sun M, Zheng Y, et al. A novel IncRNA, LUADT1, promotes lung adenocarcinoma proliferation via the epigenetic suppression of p27. Cell Death Dis. 2015;6:e1858.

7. Zheng $X$, Zhang J, Fang T, Wang X, Wang S, Ma Z, et al. The long non-coding RNA PIK3CD-AS2 promotes lung adenocarcinoma progression via YBX1-mediated suppression of p53 pathway. Oncogenesis. 2020;9:34.

8. Cao Y, Di X, Zhang Q, Li R, Wang K. RBM10 Regulates Tumor Apoptosis, Proliferation, and Metastasis. Front Oncol. 2021;11:603932.

9. Bursac S, Brdovcak MC, Donati G, Volarevic S. Activation of the tumor suppressor p53 upon impairment of ribosome biogenesis. Biochim Biophys Acta. 2014;1842:817-30.

10. Kamio T, Gu BW, Olson TS, Zhang Y, Mason PJ, Bessler M. Mice with a Mutation in the Mdm2 Gene That Interferes with MDM2/Ribosomal Protein Binding Develop a Defect in Erythropoiesis. PLoS One. 2016;11:e0152263.

11. Duan J, Ba Q, Wang Z, Hao M, Li X, Hu P, et al. Knockdown of ribosomal protein S7 causes developmental abnormalities via p53 dependent and independent pathways in zebrafish. Int J Biochem Cell Biol. 2011;43:1218-27.

12. Liu S, Tackmann NR, Yang J, Zhang Y. Disruption of the RP-MDM2-p53 pathway accelerates APC loss-induced colorectal tumorigenesis. Oncogene. 2017;36:1374-83.

13. Zhang Y, Wang J, Yuan Y, Zhang W, Guan W, Wu Z, et al. Negative regulation of HDM2 to attenuate p53 degradation by ribosomal protein L26. Nucleic acids research. 2010;38(19):6544-54.

14. Cao B, Fang Z, Liao P, Zhou X, Xiong J, Zeng S, Lu H. Cancer-mutated ribosome protein L22 (RPL22/eL22) suppresses cancer cell survival by blocking p53-MDM2 circuit. Oncotarget. 2017;8(53):90651-61.

15. Wilson-Edell KA, Kehasse A, Scott GK, Yau C, Rothschild DE, Schilling B, et al. RPL24: a potential therapeutic target whose depletion or acetylation inhibits polysome assembly and cancer cell growth. Oncotarget. 2014;5:5165-76.

16. Llanos S, Serrano M. Depletion of ribosomal protein L37 occurs in response to DNA damage and activates p53 through the L11/MDM2 pathway Cell Cycle. 2010; 9: 4005-4012. 
17. Zhou X, Hao Q, Liao J, Zhang Q, Lu H. Ribosomal protein S14 unties the MDM2-p53 loop upon ribosomal stress. Oncogene. 2013;32:388-96.

18. He X, Li Y, Dai MS, Sun XX. Ribosomal protein L4 is a novel regulator of the MDM2-p53 loop. Oncotarget. 2016;7:16217-26.

19. James A, Wang Y, Raje H, Rosby R, DiMario P. Nucleolar stress with and without p53. Nucleus. 2014;5:402-26.

20. Zou H, Chen H, Zhou Z, Wan Y, Liu Z. ATXN3 promotes breast cancer metastasis by deubiquitinating KLF4. Cancer Lett. 2019;467:19-28.

21. Watanabe S, Fujiyama H, Takafuji T, Kayama K, Matsumoto M, Nakayama KI, et al. GRWD1 regulates ribosomal protein L23 levels via the ubiquitin-proteasome system. J Cell Sci. 2018;131:jcs213009.

22. Zhao C, Tao T, Yang L, Qin Q, Wang Y, Liu H, et al. Loss of PDZK1 expression activates PI3K/AKT signaling via PTEN phosphorylation in gastric cancer. Cancer Lett. 2019;453:107-21.

23. Luo J, Liu K, Yao Y, Sun Q, Zheng X, Zhu B, et al. DMBX1 promotes tumor proliferation and regulates cell cycle progression via repressing OTX2-mediated transcription of p21 in lung adenocarcinoma cell. Cancer Lett. 2019;453:45-56.

24. Zhang $\mathrm{X}$, Wang $\mathrm{W}$, Wang $\mathrm{H}$, Wang $\mathrm{MH}$, Xu W, Zhang $\mathrm{R}$, Zhang. Identification of ribosomal protein $\mathrm{S} 25$ (RPS25)-MDM2-p53 regulatory feedback loop. Oncogene. 2013;32:2782-91.

25. Li H, He Y, Yan J, Zhao Q, Di C, Zhang H. Comparative proteomics reveals the underlying toxicological mechanism of low sperm motility induced by iron ion radiation in mice. Reprod Toxicol. 2016;65:148-58.

26. Wong CC, Traynor D, Basse N, Kay RR, Warren AJ. Defective ribosome assembly in ShwachmanDiamond syndrome. Blood. 2011;118:4305-12.

27. Shen Z, Zhou L, Zhang C, Xu J. Reduction of circular RNA Foxo3 promotes prostate cancer progression and chemoresistance to docetaxel. Cancer Lett. 2020;468:88-101.

28. Sohn EJ, Jung DB, Lee H, Han I, Lee J, Lee H, et al. CNOT2 promotes proliferation and angiogenesis via VEGF signaling in MDA-MB-231 breast cancer cells. Cancer Lett. 2018;412:88-98.

29. Song WS, Jeon YJ, Namgung B, Hong M. S. I.Yoon, A conserved TLR5 binding and activation hot spot on flagellin. Sci Rep. 2017;7:40878.

30. Cao L, Wang F, Li S, Wang X, Huang D, Jiang R. PIM1 kinase promotes cell proliferation, metastasis and tumor growth of lung adenocarcinoma by potentiating the c-MET signaling pathway. Cancer Lett. 2019;444:116-26.

31. Redman KL, Rechsteiner M. Identificati.on of the long ubiquitin extension as ribosomal protein S27a. Nature. 1989;338:438-40.

32. Hong SW, Kim SM, Jin DH, Kim YS, Hur DY. RPS27a enhances EBV-encoded LMP1-mediated proliferation and invasion by stabilizing of LMP1. Biochem Biophys Res Commun. 2017;491:303-9.

33. Wawryk-Gawda E, Chylińska-Wrzos E, Lis-Sochocka P, Chłapek M, Bulak K, Jędrych K. M, et al, P53 protein in proliferation, repair and apoptosis of cells. Protoplasma. 2014;251:525-33. 
34. Chu Y, Li D, Zhang H, Ding J, Xu P, Qiu X, Zhang H. PIG3 suppresses gastric cancer proliferation by regulating p53- mediated apoptosis. J Biol Regul Homeost Agents. 2018;32:1185-9.

35. Karimian A, Ahmadi Y, Yousefi B. Multiple functions of p21 in cell cycle, apoptosis and transcriptional regulation after DNA damage. DNA Repair. 2016;42:63-71.

36. Meng X, Franklin DA, Dong J, Zhang Y. MDM2-p53 pathway in hepatocellular carcinoma. Cancer Res. 2014;74:7161-7.

37. Jung JH, Lee H, Cao B, Liao P, Zeng SX, Lu H. RNA-binding motif protein 10 induces apoptosis and suppresses proliferation by activating $p 53$. Oncogene. 2020;39:1031-40.

38. Deisenroth C, Franklin DA, Zhang Y. Zhang The Evolution of the Ribosomal Protein-MDM2-p53 Pathway. Cold Spring Harb Perspect Med. 2016;6:a026138.

39. Cho J, Park J, Shin SC, Kim JH, Kim EE, Song EJ. Ribosomal protein S2 interplays with MDM2 to induce p53. Biochem Biophys Res Commun. 2020;523:542-7.

40. Sloan KE, Bohnsack MT, Watkins NJ. The 5S RNP couples p53 homeostasis to ribosome biogenesis and nucleolar stress. Cell Rep. 2013;5:237-47.

41. Monia BP, Ecker DJ, Jonnalagadda S, Marsh J, Gotlib L, Butt TR, et al. Gene synthesis, expression, and processing of human ubiquitin carboxyl extension proteins. J Biol Chem. 1989;264:4093-103.

42. Wang H, Yu J, Zhang L, Xiong Y, Chen S, Xing H, et al. RPS27a promotes proliferation, regulates cell cycle progression and inhibits apoptosis of leukemia cells. Biochem Biophys Res Commun. 2014;446:1204-10.

43. Fatima G, Mathan G, Kumar V. The HBx protein of hepatitis B virus regulates the expression, intracellular distribution and functions of ribosomal protein S27a. J Gen Virol. 2012;93(Pt 4):706-15.

44. Nosrati N, Kapoor NR, Kumar V. DNA damage stress induces the expression of ribosomal protein S27a gene in a p53-dependent manner. Gene. 2015;559:44-51.

45. Fumagalli S, Di Cara A, Neb-Gulati A, Natt F, Schwemberger S, Hall J, et al. Absence of nucleolar disruption after impairment of $40 \mathrm{~S}$ ribosome biogenesis reveals an rpL11-translation-dependent mechanism of p53 induction. Nat Cell Biol. 2009;11:501-8.

46. Chen L, Wang H. Nicotine Promotes Human Papillomavirus (HPV)-Immortalized Cervical Epithelial Cells (H8) Proliferation by Activating RPS27a-Mdm2-P53 Pathway In Vitro. Toxicol Sci. 2019;167:408-18.

47. Nosrati N, Kapoor NR, Kumar V. DNA damage stress induces the expression of ribosomal protein S27a gene in a p53-dependent manner. Gene. 2015;2559:44-51.

\section{Figures}


A
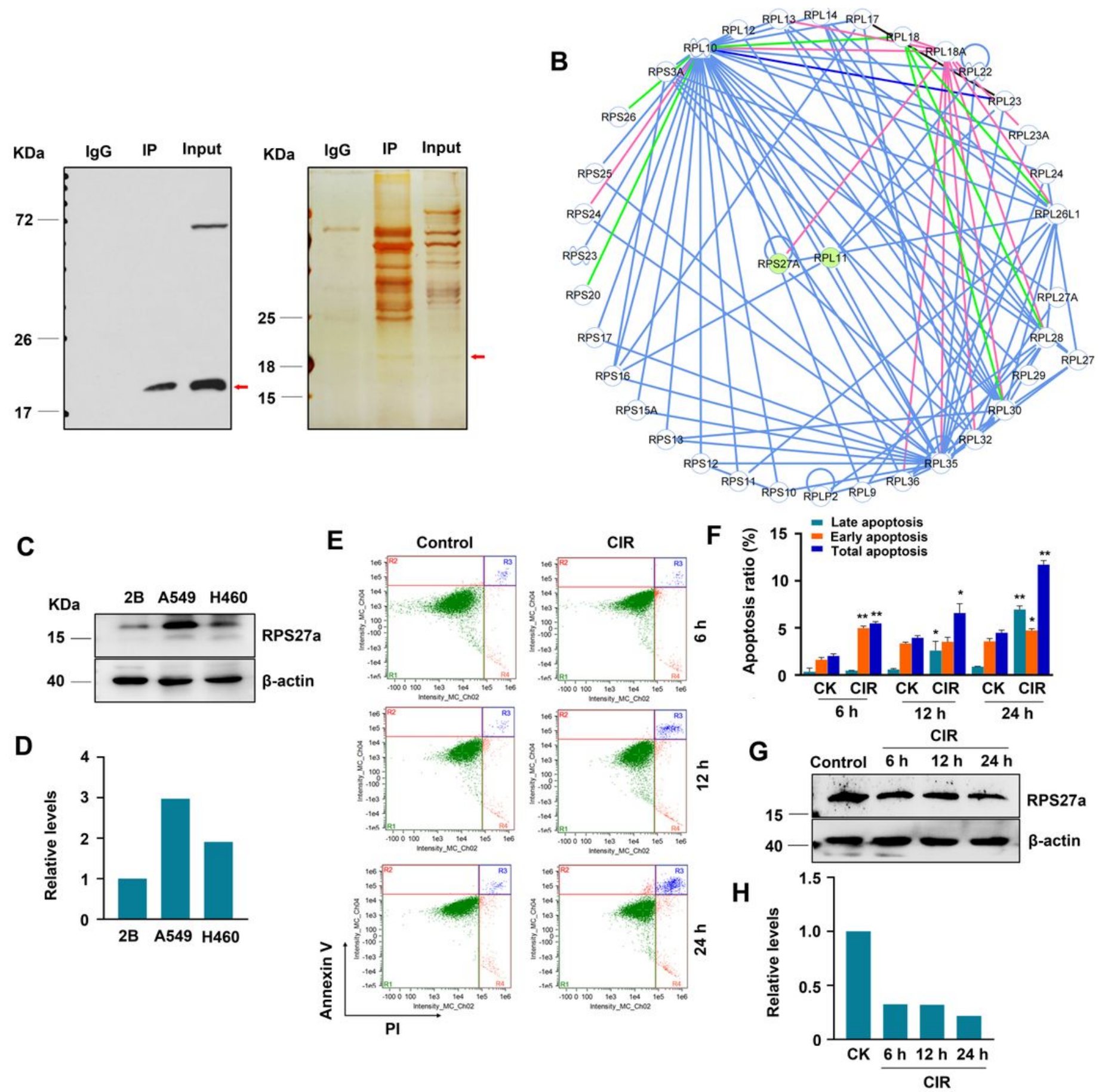

\section{Figure 1}

Identification of ribosomal proteins that interacted with RPL11. (A) The lysates of A549 cells with antiRPL11 antibody was pulled down by the endogenous RPL11-interacting protein. The immunoprecipitated proteins (IP) were separated, then stained by silver. The protein band (about $18 \mathrm{kDa}$ ), which strongly bound to RPL11, was analyzed by MALDI-TOF-MS. (B) Interaction network of ribosomal proteins with RPL11 using the STRING database; the identified combined degree of RPS27a were the highest and it might be a novel binding protein of RPL11 in A549 cells. (C) The expression of RPS27a was analyzed by immunoblotting in H460, BEAS-2B and A549 cells. (D) The expression of RPS27a in A549 and H460 cells was compared with 2B cell. (D) Apoptosis of A549 cell was analyzed by flow cytometry at 6,12 and $24 \mathrm{~h}$ 
irradiated by radiation. (E) The histograms of apoptosis were analyzed using the Student's $t$ test $\left({ }^{*} \mathrm{P}<\right.$ 0.05 , $\left.{ }^{\star *} P<0.001\right)(n=3)$ after irradiation. $(F)$ Immunoblotting analysis of RPS27a expression in A549 cells after irradiation. (G) Relative expression levels of RPS27a in irradiated groups was compared to control. CK, control; CIR, carbon ion radiation.
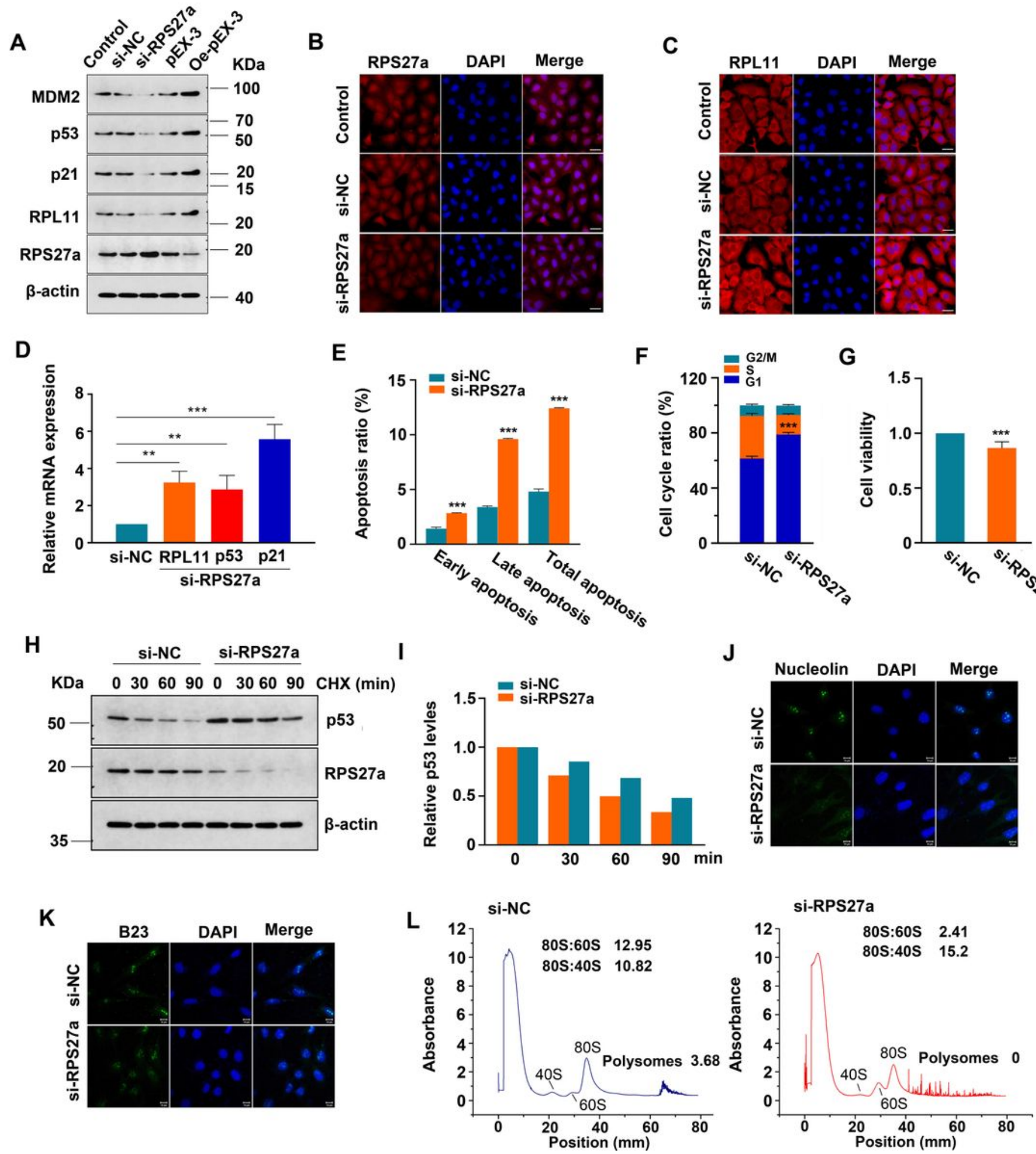

Figure 2 
Knockdown of RPS27a stabilized and activated p53, caused G1-phase arrest, induced apoptosis, and inhibited proliferation of A549 cells. (A) Knockdown of RPS27a increased the expression of p53, MDM2, RPL11 and p21. A549 cells transfected with siRNA or overexpressed plasmid ( $p E X-3)$. The expression of protein levels was analyzed by immunoblotting. (B, C) Knockdown of RPS27a increased the protein level of RPL11. A549 cells were immunofluorescence stained with RPL11 and RPS27a antibodies, DAPI staining showed the nuclei $(400 \times$ magnification, bar $=50 \mu \mathrm{m})$. (D) Knockdown of RPS27a increased the expression of genes. Statistical analysis was performed using one-way analysis of variance (ANOVA) with Turkey $\left({ }^{*} P<0.05 ; * * P<0.001\right)(n=3) .(E, F)$ Knockdown of RPS27a increased apoptosis and G1 phase arrest in A549 cells. The histograms of apoptosis and cell cycle were analyzed using the Student's $t$ test $(* \star * P<0.001)(n=3)$ after 48-h transfection. (G) Knockdown of RPS27a inhibited A549 cell proliferation. A549 cells were transfected for $48 \mathrm{~h}$ and then analyzed. Statistical analysis was performed using the Student's t test $(\star \star \star P<0.001)$. $(H, I)$ Knockdown of RPS27a extended the half-life of $p 53$. A549 cells were transfected for $48 \mathrm{~h}$ and treated with $50 \mu \mathrm{g} / \mathrm{mL}$ cycloheximide (CHX) for 30, 60, and 90 min, and the expression of p53 and RPS27a protein was analyzed by immunoblotting. (J, K) Immunofluorescence analysis of the location of Nuclelolin and B23 in A549 cells with RPS27a knockdown; the cells were fixed with $4 \%$ paraformaldehyde, stained, and observed and photographed using an immunofluorescence microscope (magnification, 400x, bar = $10 \mu \mathrm{m}$ ). (L) Polysome profile and ribosomal stress analysis. Sucrose gradient sedimentation was used to analyze the ribosomal profiles; the value of ribosomal sedimentation was measured by monitoring A254. Peaks showing 40S, 60S, 80S, and polysome contents are indicated. NC, negative control; Oe, overexpressed; $\mathrm{CHX}$, cycloheximide. 
A
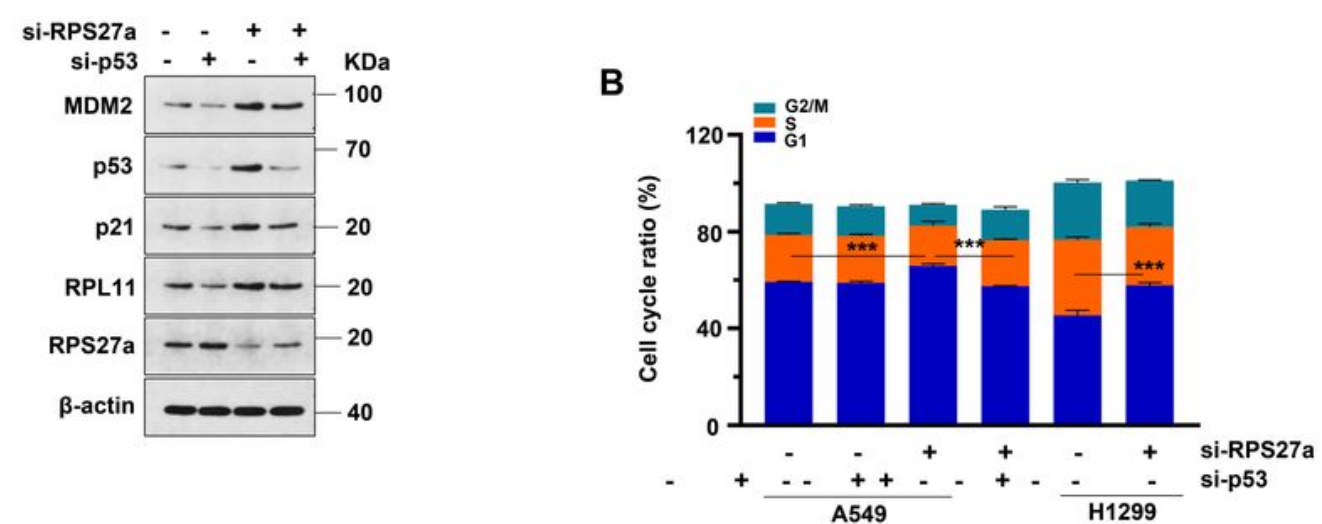

C

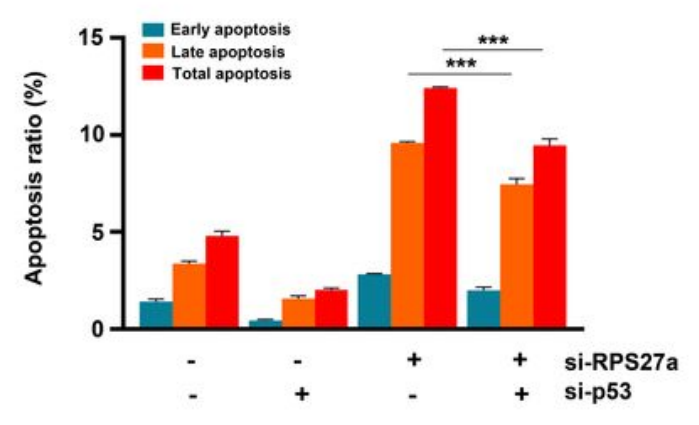

D

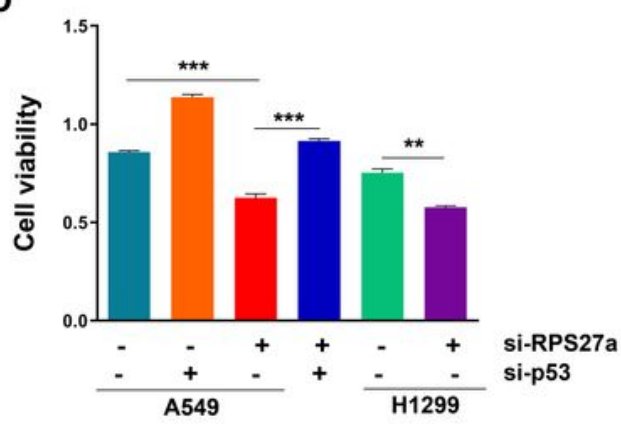

E
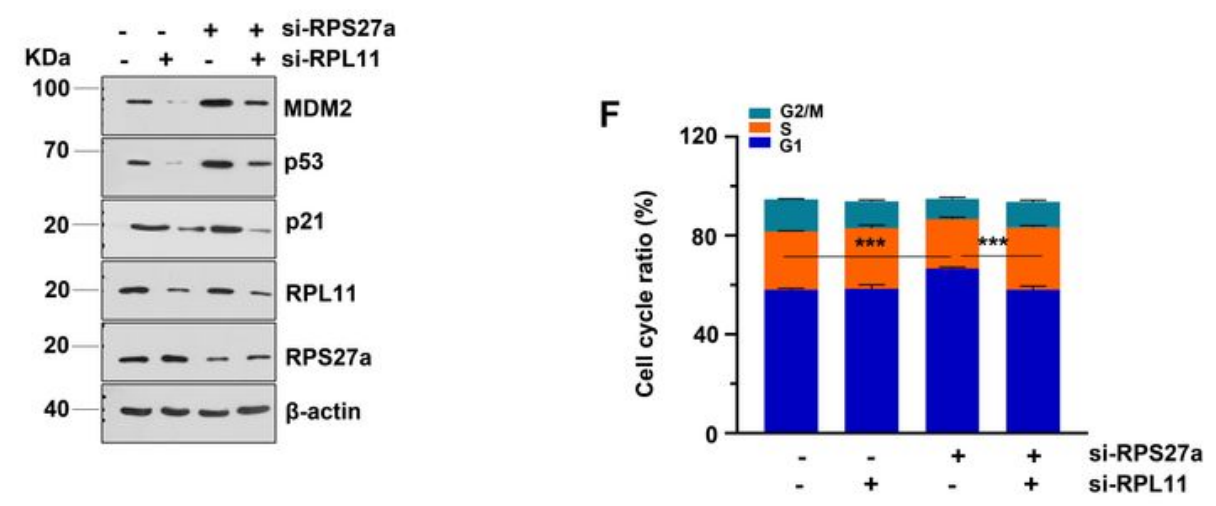

G
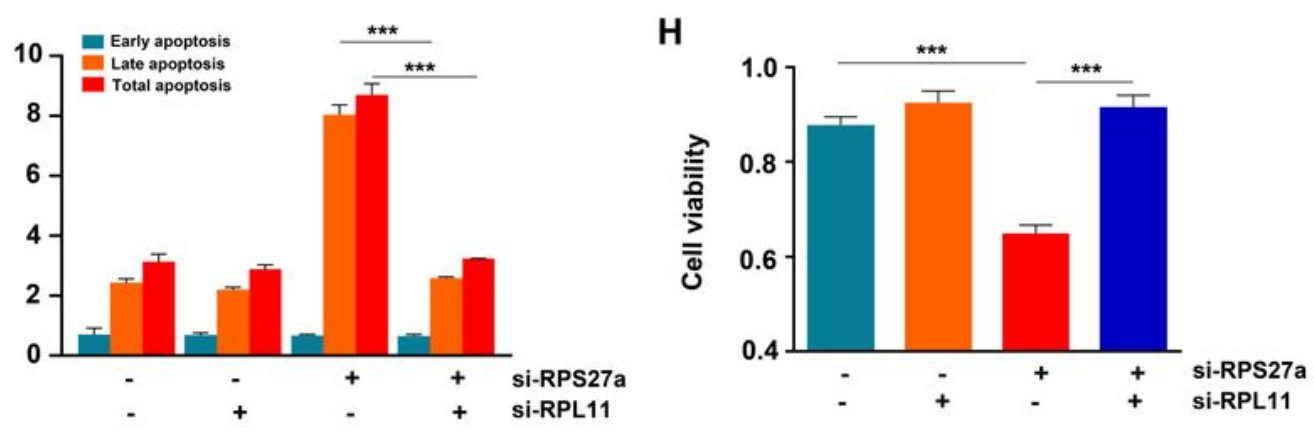

\section{Figure 3}

Knockdown of RPS27a in A549 cells induced arrest of G1 phase, which was p53 dependent; it activated p53, which was RPL11 dependent. (A) Immunoblotting showed that the knockdown of p53 eliminated the increase in p53 and p21 expression by knocking down RPS27a. (B) Histograms of the percentage $(n=3)$ of the cell cycle in A549 and H1299 cells after p53 co-transfected with RPS27a. (C) Histograms of the apoptosis ratio in A549 cells after co-transfection p53 and RPS27a; error bars represent means \pm SD ( $n=$ 
3). (D) Histograms of the proliferation in A549 and H1299 cells using the CCK-8 assay $(n=5)$. (E) Immunoblotting showed that the RPL11 knockdown eliminated the increase of p53 and p21 expression by knocking down RPS27a. (F) Histograms of the percentage $(n=3)$ of the cell cycle in A549 cells after co-transfection RPL11 and RPS27a. (G) Histograms of the apoptosis ratio $(n=3)$ in A549 cells after cotransfection RPL11 and RPS27a. $(H)$ Histograms of the proliferation $(n=5)$ in A549 cells using CCK-8 assay $(\star \star \star P<0.001)$.

A

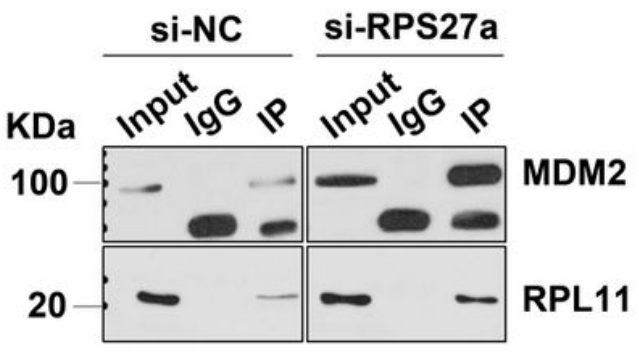

C

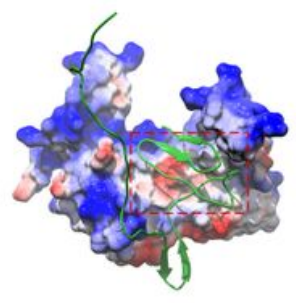

RPS27a

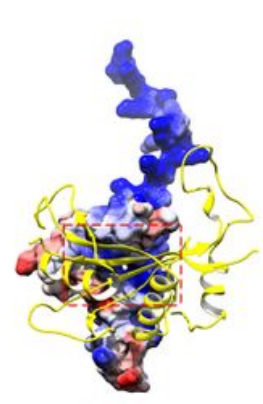

RPL11
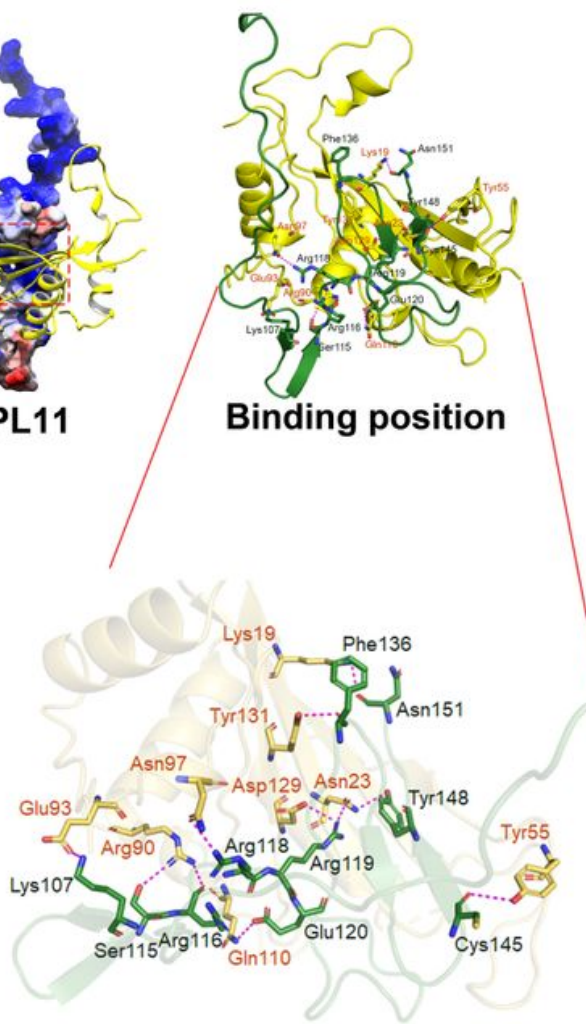

B
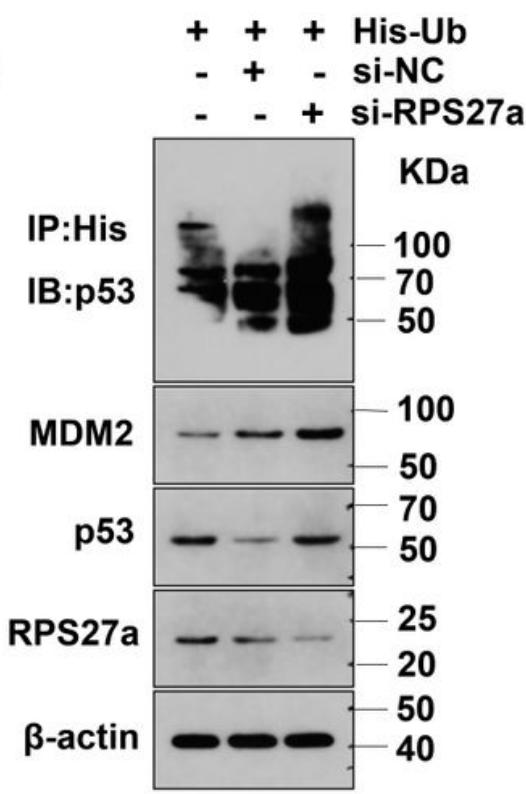

D

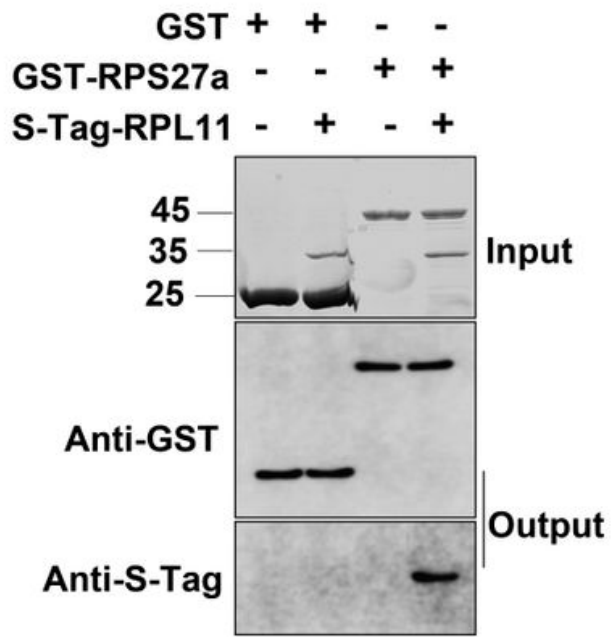

Figure 4

RPS27a directly bound to RPL11 and formed an RPS27a-RPL11-MDM2-p53 complex. (A) Knockdown of RPS27a enhanced the binding of RPL11 to MDM2. A549 cells were transfected with siRNA for $48 \mathrm{~h}$ before harvesting. (B) Knockdown of RPS27a inhibited MDM2-mediated p53 ubiquitination in vivo. A549 cells transfected with siRNA and plasmids, then treated with MG132 for $4 \mathrm{~h}$. (C) Docked position of 
RPS27a and RPL11 is shown as a cartoon model in light green and yellow. The ligands are represented as stick models in magenta, cyan and yellow-green. RPL11 and PRS27a interactions were simulated using the protein-protein interaction module of Schrodinger software (Schrodinger 2015 suit). The threedimensional crystal structures of the human 80 S ribosome (PDBID: 4v6x) were extracted from the PDB database. (D) Binding analysis of RPS27a and RPL11 in vitro by GST pull-down assays. The fusion protein beads were detected by immunoblotting using anti-GST and anti-S-Tag antibodies; GST-RPS27a and GST proteins were stained with Coomassie. NC, negative control; IP, immunoprecipitation.
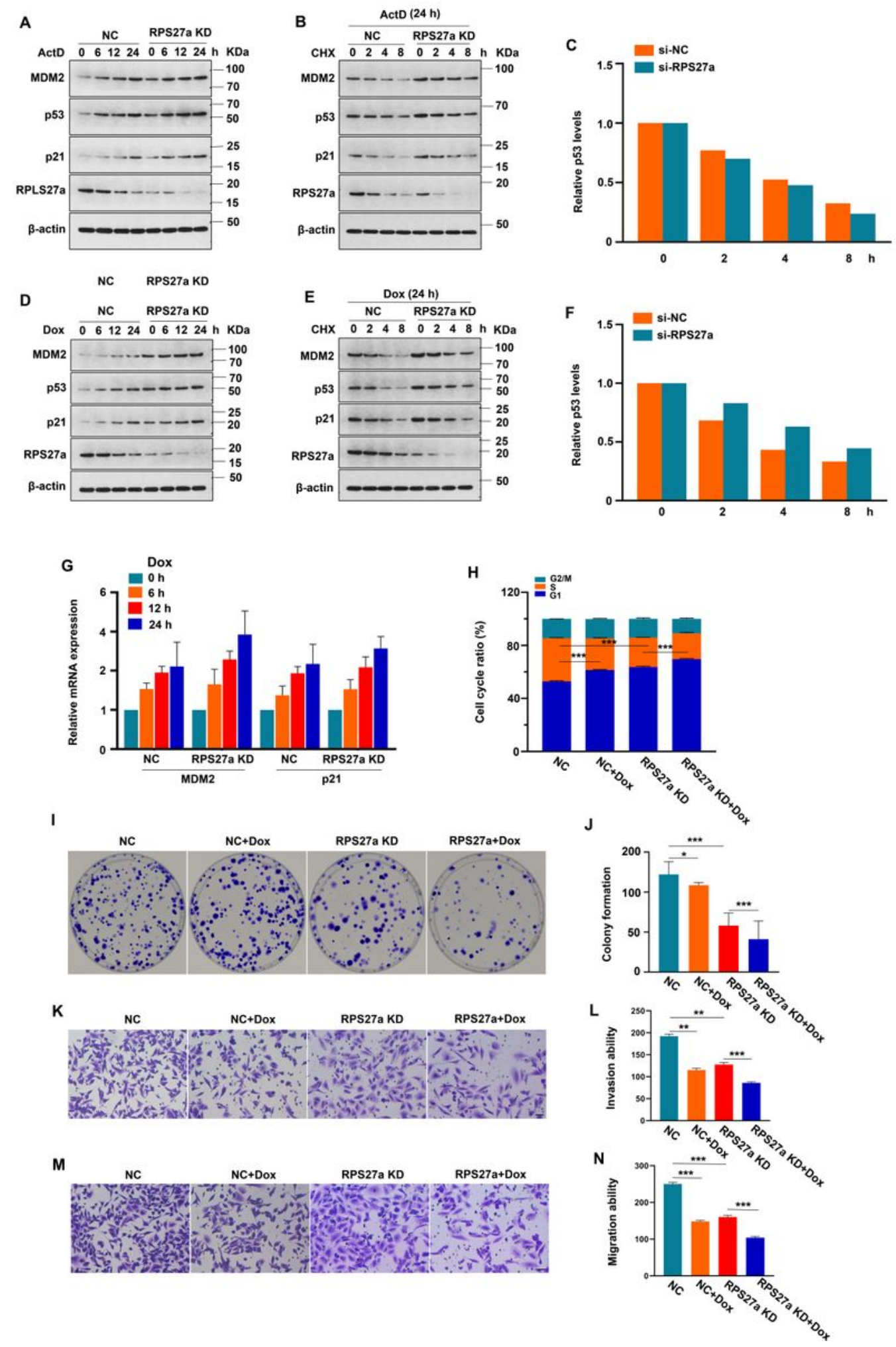


\section{Figure 5}

RPS27a did not affect p53 stability in response to ribosomal stress. (A) The ablation of RPS26 slightly reduced ActD-induced increase of p21 and MDM2. The cells with stable knockdown of RPS27a were constructed and treated with $5 \mathrm{nM}$ ActD. (B) The ablation of RPS27a did not impair p53 stabilization inducd by $5 \mathrm{nM}$ ActD. A549 cells were treated with $50 \mu \mathrm{g} / \mathrm{mL} \mathrm{CHX}$ for the indicated time periods, and then treated with ActD for a total of $24 \mathrm{~h}$. (C) The expression of p53 was quantified (p53/ $\beta$-actin), and normalized at time 0 to set at 1.0. (D) The ablation of RPS27a did not impair the upregulation of MDM2 and p21 induced by $30 \mathrm{nM}$ Dox. The cells with stable knockdown of RPS27a were treated with Dox, and then analyzed the protein expression. (E) The ablation of RPS27a did not impair p53 stabilization inducd by Dox. A549 cells were treated with $50 \mu \mathrm{g} / \mathrm{mL} \mathrm{CHX}$ for the indicated time periods, and then treated with $30 \mathrm{nM}$ Dox for a total of $24 \mathrm{~h}$. (F) The expression of p53 was quantified (p53/ $\beta$-actin), and normalized at time 0 to set at 1.0. (G) The ablation of RPS27a promoted Dox-induced expression of genes $(n=3)$. $(H)$ The ablation of RPS27a promoted $30 \mathrm{nM}$ Dox-induced cell cycle arrest $(n=5)$. $(\mathrm{I}, \mathrm{J})$ The ablation of RPS27a promoted Dox-inhibited colony formation. $(K, L)$ The ablation of RPS27a promoted Dox-inhibited invasion. (M, N) Knockdown of RPS27a promoted Dox-inhibited migration. The cell line with stable knockdown of RPS27a and control cells was treated with $30 \mathrm{nM}$ Dox for $24 \mathrm{~h}(\mathrm{n}=3)$. One-way analysis of variance (ANOVA) with Turkey was used for Statistical analysis ( $* \star * P<0.001)$. NC, negative control; ActD, Dactinomycin; Dox, Doxorubicin. 
A

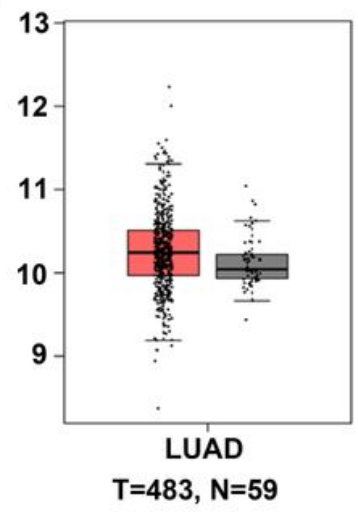

B

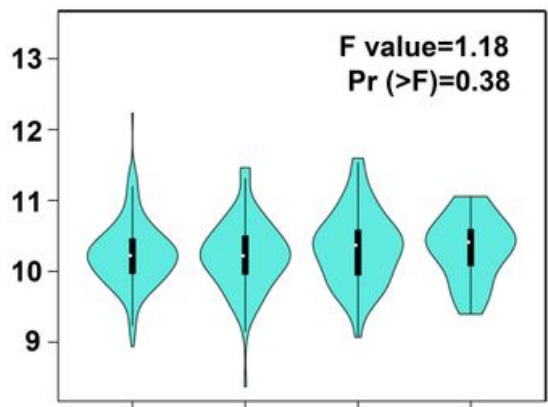

Stage I Stage II Stage III Stage IV
C

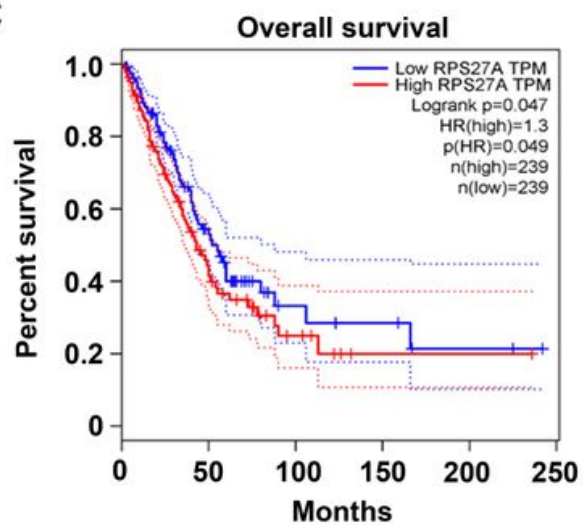

D

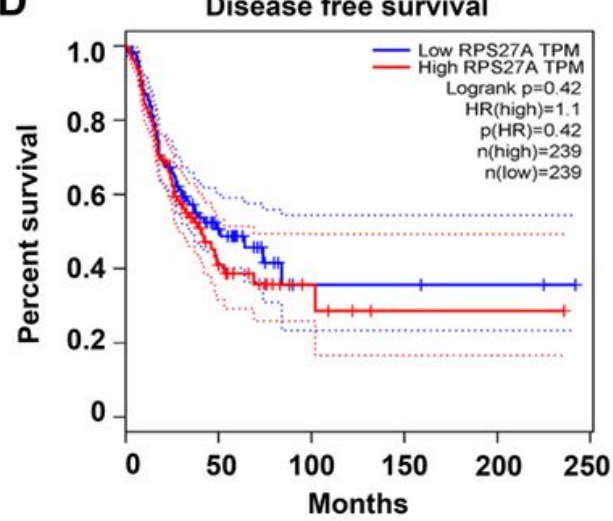

$\mathbf{F}$

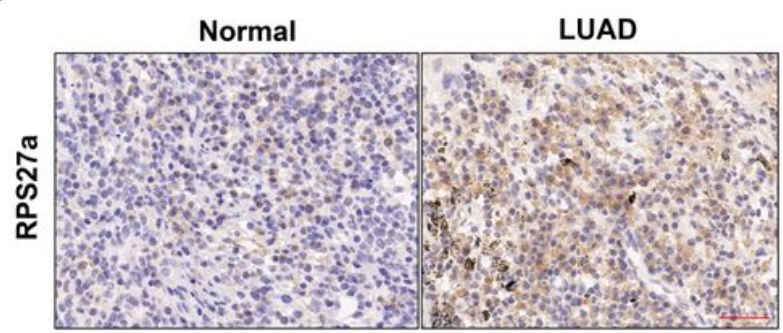

E

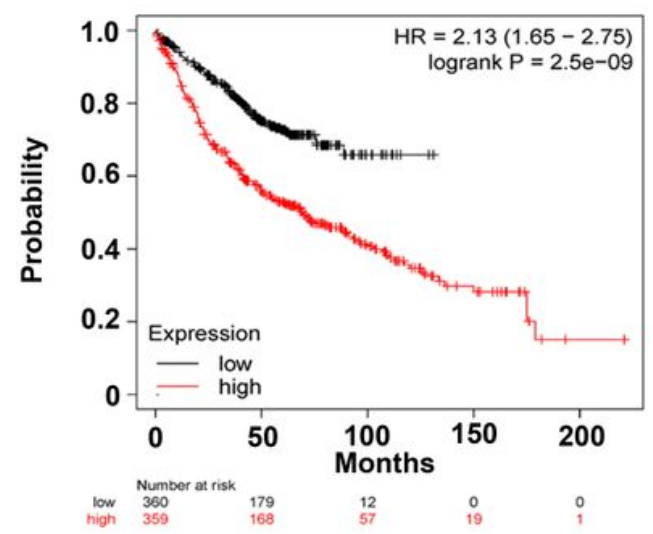

G

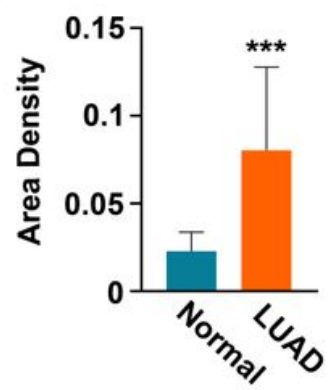

\section{Figure 6}

High levels of RPS27a correlated with LUAD. (A) The RPS27a is over-expressed in LUAD tissues. (B) GEPIA data showed that RPS27a had no difference in LUAD types of different stages (http://gepia.cancer-pku.cn/). (C) The higher overall survival rate of patients with LUAD having low RPS27a. (D) Patients with LUAD having low RPS27a expression had a higher disease-free survival rate compared with patients having high RPS27a expression. (E) High expression of RPS27a was more likely associated with cancer risk in LUAD based on K-M plotter analysis. (F) Representative images of RPS27a immunohistochemistry in patients with LUAD (magnification, 400x, bar = $50 \mu \mathrm{m}$ ). (G) RPS27a protein had significantly high expression in patients with LUAD. The quantification proteins (IOD/area) by digital image analysis, ${ }^{\star * *} \mathrm{P}<0.001$ with Student's t-test analysis. LUAD, Lung adenocarcinoma. 
A

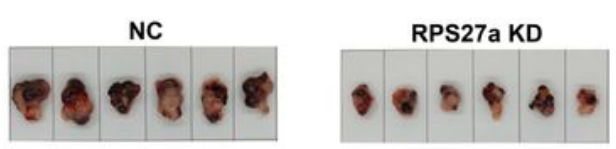

B

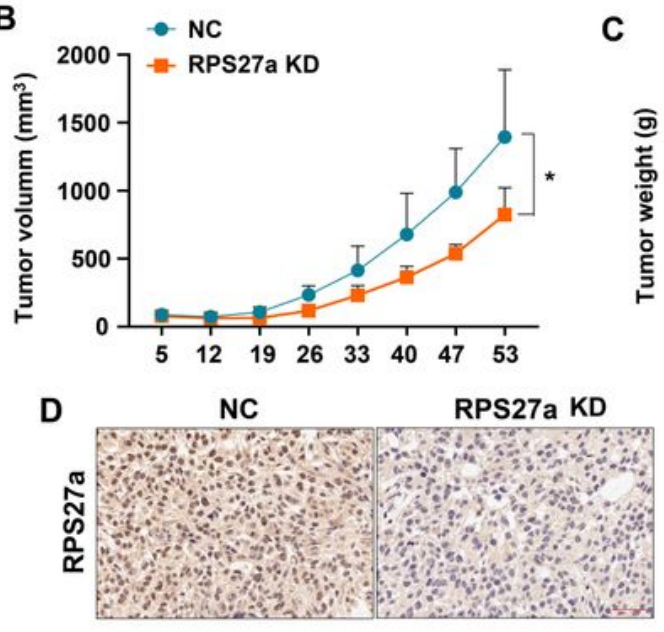

F

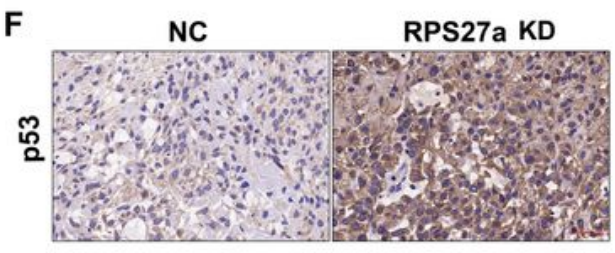

H

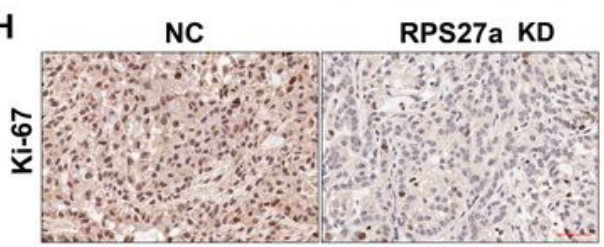

J

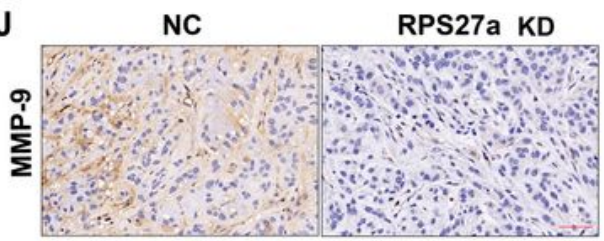

L

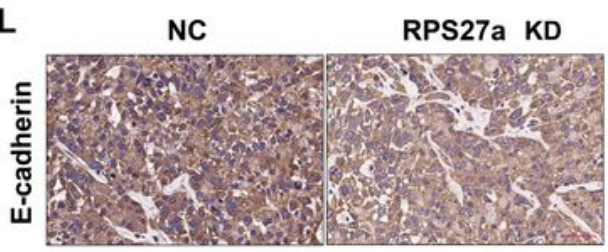

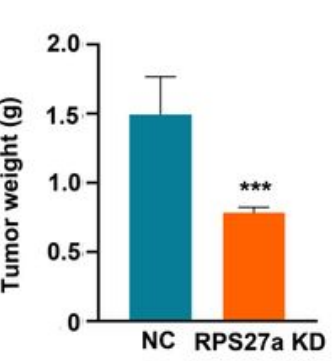

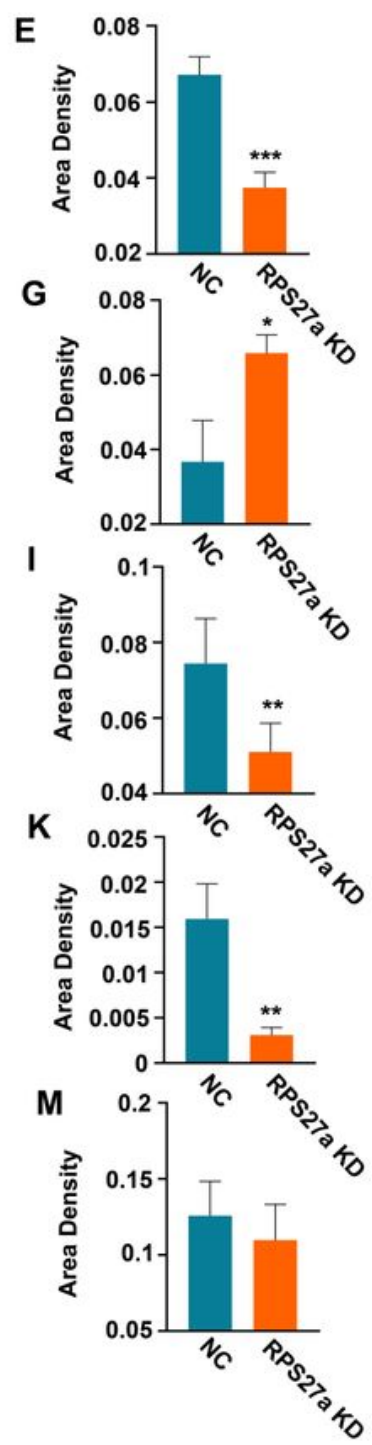

\section{Figure 7}

Knockdown of RPS27a activated p53 and inhibited growth of xenograft tumors of A549 cells. (A) Images of tumors with stable knockdown of RPS27a or control cells. The mice were sacrificed 53 days after A549 cell implantation. (B) Images of the tumor weight of sh-RPS27a or control cell xenografts. (C) Growth curves of subcutaneous xenograft tumors from stable knockdown of RPS27a or control cells. The tumor size was calculated every 7 days from 5 days after implantation, $\star \star \star P<0.001$ with Student's t-test 
analysis $(n=6)$. ( $D, F, H, J, L)$ Immunohistochemistry analysis of tumors showed relatively weak staining of RPS27a, Ki67, MMP-9, and E-cadherin, but a higher expression of p53 in stable knockdown of RPS27a cell xenograft tumors, scale bars $=50 \mu \mathrm{m}$ ( $\times 400$ magnification). $(E, G, I, K, M)$ The quantification proteins (IOD/area) by digital image analysis, ${ }^{*} P<0.05,{ }^{\star *} \mathrm{P}<0.01,{ }^{\star} * \star \mathrm{P}<0.001$ with Student's t-test analysis $(n=6)$. LUAD, Lung adenocarcinoma; NC, negative control; KD, knockdown.

\section{Supplementary Files}

This is a list of supplementary files associated with this preprint. Click to download.

- FigureS1.doc

- Figures2.doc

- FigureS3.doc

- FigureS4.doc

- Figures5.doc

- Figures6.doc

- Graphicalabstracts.jpg

- SupplementaryFile1.doc

- SupplementaryFile2.xls

- SupplementaryFile3.xls

- SupplementaryFile4.xls 